PROCEEDINGS OF THE

AMERICAN MATHEMATICAL SOCIETY

Volume 133, Number 2, Pages 605-611

S 0002-9939(04)07376-9

Article electronically published on August 25, 2004

\title{
SPACES ON WHICH EVERY POINTWISE CONVERGENT SERIES OF CONTINUOUS FUNCTIONS CONVERGES PSEUDO-NORMALLY
}

\author{
LEV BUKOVSKÝ AND KRZYSZTOF CIESIELSKI
}

(Communicated by Alan Dow)

\begin{abstract}
A topological space $X$ is a $\Sigma \Sigma^{*}$-space provided that, for every sequence $\left\langle f_{n}\right\rangle_{n=0}^{\infty}$ of continuous functions from $X$ to $\mathbb{R}$, if the series $\sum_{n=0}^{\infty}\left|f_{n}\right|$ converges pointwise, then it converges pseudo-normally. We show that every regular Lindelöf $\Sigma \Sigma^{*}$-space has the Rothberger property. We also construct, under the continuum hypothesis, a $\Sigma \Sigma^{*}$-subset of $\mathbb{R}$ of cardinality continuum.
\end{abstract}

\section{INTRODUCTION}

We will use standard set-theoretical notation as in 4. In particular, the ordinal numbers will be identified with the sets of their predecessors and cardinals with the initial ordinals. Thus, $2=\{0,1\}$ and the first infinite ordinal number $\omega$ is equal to the set of all natural numbers $\{0,1,2, \ldots\}$. The family of all functions from a set $X$ into $Y$ is denoted by $Y^{X}$. Thus, for $n<\omega$ symbol $2^{n}$ will stand for the set of all sequences $s:\{0,1,2, \ldots, n-1\} \rightarrow\{0,1\}$, while $2^{<\omega}=\bigcup_{n<\omega} 2^{n}$ is the set of all finite sequences into 2. For a set $X$ and a cardinal number $\kappa$ we define $[X]^{\kappa}$ as the family of all subsets of $X$ of cardinality $\kappa$. Families $[X]^{<\kappa}$ and $[X]^{\leq \kappa}$ are defined similarly. We let $\mathfrak{C} \subset[0,1]$ be the classical Cantor middle-thirds set. For a topological space $X$ we write $\mathcal{C}(X)$ for the family of continuous functions from $X$ into the set $\mathbb{R}$ of real numbers. For a sequence $\left\langle f_{n}\right\rangle_{n<\omega}$ of continuous functions from $X$ into $\mathbb{R}$ we let

$$
S\left(\left\langle f_{n}\right\rangle\right)=\left\{x \in X: \sum_{n<\omega}\left|f_{n}(x)\right|<\infty\right\} .
$$

A series $\sum_{n<\omega} f_{n}$ of functions from a set $X$ into $\mathbb{R}$ converges normally (respectively, pseudo-normally) on a set $A \subset X$ if there exists a sequence $\left\langle\varepsilon_{n}: n<\omega\right\rangle$ of positive reals such that $\sum_{n<\omega} \varepsilon_{n}<\infty$ and for every $x \in A$ we have $\left|f_{n}(x)\right| \leq \varepsilon_{n}$ for all (respectively, all but finitely many) $n<\omega$. Thus, a topological space $X$ belongs to $\Sigma \Sigma^{*}$ (see [3]) provided that, for any sequence $\left\langle f_{n} \in \mathcal{C}(X): n<\omega\right\rangle$ with $S\left(\left\langle f_{n}\right\rangle\right)=$ $X$, the series $\sum_{n<\omega} f_{n}$ converges pseudo-normally on $X$.

Received by the editors January 8, 2003 and, in revised form, June 5, 2003.

2000 Mathematics Subject Classification. Primary 54G99, 03E35; Secondary 54A35, 54C30.

Key words and phrases. $\Sigma \Sigma^{*}$-space, Rothberger property, quasinormal convergence, pseudonormal convergence.

This work was partially supported by NATO Grant PST.CLG.977652. The second author was also supported by 2002/03 West Virginia University Senate Research Grant.

(C)2004 American Mathematical Society 
The class $\Sigma \Sigma^{*}$ has been studied, in a context of several similar classes of sets, by Bukovský, Recław, and Repický in [3]. In particular, it is known (see [3] Diagram 2]) that every $\Sigma \Sigma^{*}$-space $X$ is a $\sigma$-space, that is, every $G_{\delta}$ subset of $X$ is also an $F_{\sigma}$ subset of $X$. One of the main results of this paper says that Lindelöf $\Sigma \Sigma^{*}$-spaces are small also in a different sense.

Theorem 1. Every regular and Lindelöf $\Sigma \Sigma^{*}$-space has the Rothberger property.

Recall here that a topological space $X$ has the Rothberger property (called sometimes property $C^{\prime \prime}$ ) provided that, for every sequence $\left\langle\mathcal{G}_{n}: n<\omega\right\rangle$ of open covers of $X$, we can find $U_{n} \in \mathcal{G}_{n}$ for every $n<\omega$ such that $\left\{U_{n}: n<\omega\right\}$ covers $X$.

Certainly the most interesting case of Theorem 1 is when the space is separable and metric. It should be stressed that although many classes from [3, Diagram 2] are contained in the class of $\sigma$-spaces, within the class of separable metric spaces only $\Sigma \Sigma^{*}$-spaces have the Rothberger property. Recall also that every metric space $X$ with the Rothberger property is of strong measure zero. (See e.g. [1, thm. 8.1.11].) Since there are models of ZFC in which every strong measure zero subset of $\mathbb{R}$ is countable [8, thm. 3.2], an uncountable $\Sigma \Sigma^{*}$-subset of $\mathbb{R}$ cannot be constructed in ZFC. Moreover, so far there has been no consistent example of a $\Sigma \Sigma^{*}$-subset of $\mathbb{R}$ of cardinality continuum. This state changes with the following theorem.

Theorem 2. If the continuum hypothesis holds then there exists a set $X \subset[0,1]$ of cardinality continuum that belongs to $\Sigma \Sigma^{*}$.

Note that the existence of such a set does not seem to follow from any other known constructions, since $\Sigma \Sigma^{*}$ does not contain any other known (to us) class for which the analogous result is known. Indeed, one of the smallest classes of sets that admits the consistent examples of cardinality continuum subsets of $\mathbb{R}$ is the class of strong $\gamma$-sets 1 (See [5].) However, there are consistent examples of strong $\gamma$-sets that are not in $\Sigma \Sigma^{*}$. Actually, slightly modifying the proof of [5, thm. 8] it is easy to construct, under Martin's axiom, a strong $\gamma$-subset of $\mathbb{R}$ of cardinality continuum that is continuum-concentrated on a countable subset. By theorems 3.12 and 4.1 from [3], such a set is not in $\Sigma \Sigma^{*}$.

\section{Every Regular LindelöF $\Sigma \Sigma^{*}$-SPACE HaS the Rothberger PROPERTY}

We start with the following variation of a well-known characterization of the Rothberger property.

Lemma 3. Let $\left\langle m_{n}\right\rangle_{n<\omega}$ be a sequence of positive integers. Then a topological space $X$ has the Rothberger property if and only if

(*) for every sequence $\left\langle\mathcal{U}_{n}\right\rangle_{n<\omega}$ of open covers of $X$ there exist an increasing sequence $\left\langle n_{i}<\omega: i<\omega\right\rangle$ and sets $\mathcal{V}_{n_{i}} \subset \mathcal{U}_{n_{i}}$ with $\left|\mathcal{V}_{n_{i}}\right| \leq m_{n_{i}}$ such that $X=\bigcup_{i<\omega} \bigcup \mathcal{V}_{n_{i}}$.

Proof. Clearly every space with the Rothberger property satisfies $(*)$.

To see the other implication let $\left\langle\mathcal{G}_{n}: n<\omega\right\rangle$ be a sequence of open covers of $X$. For $n<\omega$ put $p_{n}=\sum_{k=0}^{n} m_{k}$ and let $\mathcal{U}_{n}$ be an open cover of $X$ refining covers $\mathcal{G}_{k}$ for $k<p_{n}$. Let also $p_{-1}=0$. Applying $(*)$ to $\left\langle\mathcal{U}_{n}\right\rangle_{n<\omega}$ we can find appropriate sequences $\left\langle n_{i}\right\rangle_{i<\omega}$ and $\left\langle\mathcal{V}_{n_{i}}\right\rangle_{i<\omega}$. For $n<\omega$ let $\mathcal{W}_{n}=\left\{W_{k} \in \mathcal{U}_{n}: p_{n-1} \leq k<p_{n}\right\}$

\footnotetext{
${ }^{1}$ Every strong $\gamma$-set has the $\gamma$-property, which is the strongest property in Scheepers' diagram [7] admitting consistent examples of cardinality continuum.
} 
be such that $\mathcal{V}_{n_{i}} \subset \mathcal{W}_{n_{i}}$ for every $i<\omega$. (Thus, for $n$ not equal to any $n_{i}$ the sets in $\mathcal{W}_{n}$ are arbitrary.) Then we have $\bigcup_{k<\omega} W_{k} \supset \bigcup_{i<\omega} \bigcup \mathcal{V}_{n_{i}}=X$. But for $p_{n-1} \leq k<p_{n}$ the set $W_{k}$ belongs to $\mathcal{U}_{n}$, which is a refinement of $\mathcal{G}_{k}$. So, we can find $U_{k} \in \mathcal{G}_{k}$ for which $W_{k} \subset U_{k}$. Therefore, $X=\bigcup_{k<\omega} U_{k}$.

Proof of Theorem 1]. Since $X$ is regular and Lindelöf, it is completely regular. Thus, being a $\Sigma \Sigma^{*}$-space, $X$ must be zero-dimensional - see [2, cor. 4.5]. Let $\left\langle\mathcal{U}_{n}\right\rangle_{n<\omega}$ be a sequence of open covers of $X$. We will show that the condition $(*)$ from Lemma 3 is fulfilled.

Since $X$ is Lindelöf and zero-dimensional we can assume, replacing with a refinement, if necessary, that each cover $\mathcal{U}_{n}$ is countable and consists of pairwise disjoint clopen sets. For every $n<\omega$ let $\left\{U_{k}: k \in P_{n}\right\}$ be an enumeration of $\mathcal{U}_{n}$, where $\left\{P_{n}: n<\omega\right\}$ is an appropriate partition of $\omega$.

Let $\left\langle m_{n}\right\rangle_{n<\omega}$ be a sequence of positive integers such that $r=\sum_{n=0}^{\infty} 1 / m_{n}<\infty$. For every $n<\omega$ and $k \in P_{n}$ let $f_{k}=\frac{1}{m_{n}} \chi_{U_{k}}$, where $\chi_{U}: X \rightarrow\{0,1\}$ is the characteristic function of $U$. Then for every $x \in X$

$$
\sum_{k<\omega} f_{k}(x)=\sum_{n<\omega} \sum_{k \in P_{n}} f_{k}(x)=\sum_{n<\omega} \frac{1}{m_{n}}=r .
$$

Since $X$ is a $\Sigma \Sigma^{*}$-space, there exists a sequence $\left\langle\varepsilon_{n}: n<\omega\right\rangle$ of positive reals such that $\sum_{n<\omega} \varepsilon_{n}<\infty$, and for every $x \in X$ we have $f_{n}(x) \leq \varepsilon_{n}$ for all but finitely many $n<\omega$. For $i, n<\omega$ let $X_{i}=\left\{x \in X: f_{k}(x) \leq \varepsilon_{k}\right.$ for all $\left.i \leq k<\omega\right\}$ and put $T_{i}^{n}=\left\{k \in P_{n}: X_{i} \cap U_{k} \neq \emptyset\right\}$. We claim that for any $i<\omega$

$$
\text { there exist infinitely many } n \in \omega \text { such that }\left|T_{i}^{n}\right| \leq m_{n} \text {. }
$$

To see this, by way of contradiction assume that there is an $i<\omega$ for which (1) is false. So, there exists an $n_{0}$ such that $\left|T_{i}^{n}\right|>m_{n}$ for each $n \geq n_{0}$. Moreover, increasing $n_{0}$, if necessary, we can assume that $k \geq i$ for any $k \in T_{i}^{n}$ and $n \geq n_{0}$. Hence for every $k \in T_{i}^{n}$ and $n \geq n_{0}$ there is an $x \in \bar{X}_{i} \cap U_{k}$, and so $\frac{1}{m_{n}}=f_{k}(x) \leq \varepsilon_{k}$. Consequently $\sum_{k=0}^{\infty} \varepsilon_{k} \geq \sum_{n \geq n_{0}} \sum_{k \in T_{i}^{n}} \varepsilon_{k} \geq \sum_{n \geq n_{0}}\left|T_{i}^{n}\right| \cdot 1 / m_{n}=\infty$, which is a contradiction.

Now let $\left\langle n_{i}<\omega: i<\omega\right\rangle$ be an increasing sequence such that $\left|T_{i}^{n_{i}}\right| \leq m_{n_{i}}$. Setting $\mathcal{V}_{n_{i}}=\left\{U_{j}^{n_{i}}: j \in T_{i}^{n_{i}}\right\} \subset \mathcal{U}_{n_{i}}$ we obtain a cover satisfying (*) from Lemma 3

\section{3. $\Sigma \Sigma^{*}$-SUBSET OF $\mathbb{R}$ OF CARDINALITY CONTINUUM}

We start with recalling Egoroff's theorem (see e.g. [10, p. 73]):

Let $\mu$ be a finite countably additive measure on a set $X$ and let $\left\langle g_{k}\right\rangle_{k<\omega}$ be a pointwise convergent sequence of measurable functions from $X$ into $\mathbb{R}$. Then for every $\varepsilon>0$ there exists a measurable set $E \subset X$ such that $\mu(X \backslash E)<\varepsilon$ and $\left\langle g_{k}\right\rangle_{k<\omega}$ converges uniformly on $E$.

In the proof of the theorem we will use the following result, which is of interest on its own.

Proposition 4. Let $P$ be a perfect subset of $\mathbb{R}$ and $\left\langle f_{n} \in \mathcal{C}(P): n<\omega\right\rangle$. If $S\left(\left\langle f_{n}\right\rangle\right)=P$, then there exists a sequence $\left\langle\varepsilon_{n}: n<\omega\right\rangle$ of positive numbers such that $\sum_{n<\omega} \varepsilon_{n}<\infty$ and the closed set $K=\bigcap_{n<\omega}\left\{x \in P:\left|f_{n}(x)\right| \leq \varepsilon_{n}\right\}$ has cardinality continuum. 
Proof. For $k<\omega$ and $x \in P$ define $g_{k}(x)=\sum_{n=k}^{\infty}\left|f_{n}(x)\right|$. Then functions $g_{k}: P \rightarrow$ $\mathbb{R}$ are measurable (in fact, Baire class one) and they converge pointwise to 0 . By Egoroff's theorem (used with a countably additive Borel probability measure on $P$ that vanishes on points) there exists a perfect subset $Q$ of $P$ on which the sequence $\left\langle g_{k}\right\rangle$ converges uniformly. By induction on $k<\omega$ we will construct the sequences $\left\langle\varepsilon_{n}^{k}>0: k, n<\omega\right\rangle$ and $\left\langle x_{s} \in Q: s \in 2^{k} \& k<\omega\right\rangle$ such that for every $0<k<\omega$, $s \in 2^{k-1}, t \in 2^{k}$, and $n<\omega$ the following holds true:

(i) $x_{\emptyset} \in Q$ is arbitrary, $\varepsilon_{n}^{0}=\left|f_{n}\left(x_{\emptyset}\right)\right|+2^{-n}$; thus $\sum_{m=0}^{\infty} \varepsilon_{m}^{0}<\infty$;

(ii) $x_{s^{\wedge} 0}=x_{s}$ and $0<\left|x_{s^{\wedge} 1}-x_{s}\right|<4^{-k} \delta_{k-1}$, where the number $\delta_{k-1}$ is defined as $\min \left(\left\{\left|x_{s_{0}}-x_{s_{1}}\right|>0: s_{0}, s_{1} \in 2^{k-1}\right\} \cup\{1\}\right)$;

(iii) $\left|f_{n}\left(x_{t}\right)\right|<\varepsilon_{n}^{k}$ and $\varepsilon_{n}^{k-1} \leq \varepsilon_{n}^{k}$;

(iv) $\sum_{m=0}^{\infty} \varepsilon_{m}^{k} \leq 2^{-k}+\sum_{m=0}^{\infty} \varepsilon_{m}^{k-1}$ and $\varepsilon_{i}^{k}=\varepsilon_{i}^{i}$ for $i<k$.

First notice that if such sequences can be constructed and we put $\varepsilon_{n}=\varepsilon_{n}^{n}$, then the sequence $\left\langle\varepsilon_{n}\right\rangle_{n<\omega}$ is as desired. Indeed, the series converges, since by (iv) for every $k<\omega$ we have

$$
\sum_{m=0}^{k} \varepsilon_{m}=\sum_{m=0}^{k} \varepsilon_{m}^{m}=\sum_{m=0}^{k} \varepsilon_{m}^{k} \leq \sum_{m=0}^{\infty} \varepsilon_{m}^{k} \leq \sum_{m=1}^{k} 2^{-m}+\sum_{m=0}^{\infty} \varepsilon_{m}^{0}<1+\sum_{m=0}^{\infty} \varepsilon_{m}^{0},
$$

so $\sum_{m=0}^{\infty} \varepsilon_{m} \leq 1+\sum_{m=0}^{\infty} \varepsilon_{m}^{0}<\infty$.

To see that the set $K=\bigcap_{n<\omega}\left\{x \in P:\left|f_{n}(x)\right| \leq \varepsilon_{n}^{n}\right\}$ has cardinality continuum, notice that $K$ is closed and that, by conditions (iii) and (iv), we have $x_{t} \in K$ for every $t \in 2^{<\omega}$. So $K$ contains the closure of the set $\left\{x_{t}: t \in 2^{<\omega}\right\}$. But, by (ii), the mapping associating $\lim _{k \rightarrow \infty} x_{\varphi \nmid k} \in K$ to each $\varphi \in 2^{\omega}$ is one-to-one.

To make an inductive step in the construction take a $k>0$ and assume that the $(k-1)$-th step of the construction is already done. Find a $j<\omega$ such that $j>k$ and $g_{j}(x)<4^{-k}$ for all $x \in Q$. Let $0<\delta \leq 4^{-k} \delta_{k-1}$ be such that for every $s \in 2^{k-1}, n \leq j$, and $x \in Q$, if $\left|x-x_{s}\right|<\delta$, then $\left|f_{n}(x)\right|<\varepsilon_{n}^{k-1}$. Existence of such a $\delta$ follows from the inductive assumption (iii) and the continuity of functions $f_{n}$. For $s \in 2^{k-1}$ pick $x_{s^{\wedge} 1} \in Q$ such that $0<\left|x_{s^{\wedge} 1}-x_{s}\right|<\delta$. This insures (ii). For $n<j$ put $\varepsilon_{n}^{k}=\varepsilon_{n}^{k-1}$ and for $j \leq n<\omega$ define $\varepsilon_{n}^{k}=\varepsilon_{n}^{k-1}+\sum_{t \in 2^{k}}\left|f_{n}\left(x_{t}\right)\right|$. This clearly guarantees (iii) and the second part of (iv). To see the first part of (iv) notice that

$$
\begin{aligned}
\sum_{m=0}^{\infty} \varepsilon_{m}^{k} & =\sum_{m<j} \varepsilon_{m}^{k}+\sum_{m=j}^{\infty} \varepsilon_{m}^{k} \\
& =\sum_{m<j} \varepsilon_{m}^{k-1}+\sum_{m=j}^{\infty}\left(\varepsilon_{m}^{k-1}+\sum_{t \in 2^{k}}\left|f_{m}\left(x_{t}\right)\right|\right) \\
& =\sum_{t \in 2^{k}} \sum_{m=j}^{\infty}\left|f_{m}\left(x_{t}\right)\right|+\sum_{m=0}^{\infty} \varepsilon_{m}^{k-1} \\
& =\sum_{t \in 2^{k}} g_{j}\left(x_{t}\right)+\sum_{m=0}^{\infty} \varepsilon_{m}^{k-1} \\
& \leq 2^{k} 4^{-k}+\sum_{m=0}^{\infty} \varepsilon_{m}^{k-1}=2^{-k}+\sum_{m=0}^{\infty} \varepsilon_{m}^{k-1} .
\end{aligned}
$$

This finishes the proof of Proposition 4 
In Proposition 4, if we require only that the sequence $\left\langle\varepsilon_{n}: n<\omega\right\rangle$ converges to zero then, by Egoroff's theorem, we can additionally assume that the set $K$ has a positive measure. However, the set $K$ from Proposition 4 need not have positive measure.

In the proof that follows we will use the following simple fact.

Fact 5. For every $X \subset \mathbb{R}$ and $\left\langle f_{n} \in \mathcal{C}(X): n<\omega\right\rangle$ such that $S\left(\left\langle f_{n}\right\rangle\right)=X$ there exists a Borel set $B \subset \mathbb{R}$ containing $X$ and extensions $f_{n}^{*} \in \mathcal{C}(B)$ of the functions $f_{n}$ such that $S\left(\left\langle f_{n}^{*}\right\rangle\right)=B$.

Proof. For every $n<\omega$ there exists a $G_{\delta}$ set $G_{n} \subset \mathbb{R}$ containing $X$ and an extension $\hat{f}_{n} \in \mathcal{C}\left(G_{n}\right)$ of $f_{n}$. Let $G=\bigcap_{n<\omega} G_{n} \supset X$, and let us define $B$ as $\bigcup_{m<\omega} \bigcap_{k<\omega}\left\{x \in G: \sum_{n \leq k}\left|\hat{f}_{n}(x)\right| \leq m\right\}$. Then $B$ is a Borel set containing $X$ and $B=S\left(\left\langle\hat{f}_{n} \uparrow G\right\rangle\right)$. Thus functions $f_{n}^{*}=\hat{f}_{n} \uparrow B$ are as required.

Proof of Theorem 2 , Let $\mathcal{B}$ be a fixed countable clopen basis for $\mathfrak{C}$. For $G \subset \mathbb{R}$ let $\mathcal{K}(G)$ be the family of all sequences $\left\langle f_{n} \in \mathcal{C}(G): n<\omega\right\rangle$ with $S\left(\left\langle f_{n}\right\rangle\right)=G$ and let $\left\langle\left\langle f_{n}^{\xi}\right\rangle_{n<\omega}: \xi<\omega_{1}\right\rangle$ be an enumeration of the family

$$
\mathcal{K}=\bigcup\{\mathcal{K}(G): G \text { is a Borel subset of } \mathfrak{C}\}
$$

such that every $f_{n}^{0}$ is the constant zero function on $\mathfrak{C}$. By induction on $\xi<\omega_{1}$ we will construct a sequence $\left\langle\left\langle x_{\xi},\left\langle\varepsilon_{n}^{\xi}\right\rangle_{n<\omega},\left\langle P_{n}^{\xi}\right\rangle_{n<\omega}, P_{\xi}, \mathcal{G}_{\xi}\right\rangle: \xi<\omega_{1}\right\rangle$ such that $x_{0}=0$, $\varepsilon_{n}^{0}=2^{-n}, P_{0}=P_{n}^{0}=\mathfrak{C}, \mathcal{G}_{0}=\{\mathfrak{C}\}$, and the following conditions are satisfied for every $0<\xi<\omega_{1}$ and $k<\omega$ :

(a) $P_{\xi}=\bigcup_{n<\omega} P_{n}^{\xi}, 0<\varepsilon_{k}^{\xi}<\infty$, and $\sum_{n<\omega} \varepsilon_{n}^{\xi}<\infty$.

(b) If $\bigcap_{\eta<\xi} P_{\eta} \not \subset S\left(\left\langle f_{n}^{\xi}\right\rangle_{n<\omega}\right)$, then

$$
-x_{\xi} \in \bigcap_{\eta<\xi} P_{\eta} \backslash S\left(\left\langle f_{n}^{\xi}\right\rangle\right), \varepsilon_{k}^{\xi}=2^{-k} \text {, and } P_{k}^{\xi}=\mathfrak{C} ;
$$

otherwise,

$-P_{k}^{\xi}=\bigcap_{k<n<\omega}\left\{x \in G_{\xi}:\left|f_{n}^{\xi}(x)\right| \leq \varepsilon_{n}^{\xi}\right\}$, where $G_{\xi}$ is the domain of the functions $f_{n}^{\xi}, n<\omega$; $x_{\xi} \in \bigcap_{\eta \leq \xi} P_{\eta} \backslash\left\{x_{\eta}: \eta<\xi\right\}$.

(c) $\mathcal{G}_{\xi}$ is a non-empty countable family of perfect subsets of $\bigcap_{\eta \leq \xi} P_{\eta}$ such that

- if $T \in \mathcal{G}_{\xi}, U \in \mathcal{B}$, and $T \cap U \neq \emptyset$, then $T \cap U \in \mathcal{G}_{\xi}$;

- every $H \in \bigcup_{\eta<\xi} \mathcal{G}_{\eta}$ contains a subset $T$ from the family $\mathcal{G}_{\xi}$.

It should be clear that if we put $X=\left\{x_{\xi}: \xi<\omega_{1}\right\}$, then $X$ is uncountable and belongs to $\Sigma \Sigma^{*}$.

Indeed, $X$ is uncountable, since $S\left(\left\langle f_{n}^{\xi}\right\rangle_{n<\omega}\right)=\mathfrak{C} \supset \bigcap_{\eta<\xi} P_{\eta}$ for uncountably many $\xi<\omega_{1}$. To see that $X \in \Sigma \Sigma^{*}$ take a sequence $\left\langle f_{n} \in \mathcal{C}(X): n<\omega\right\rangle$ for which $S\left(\left\langle f_{n}\right\rangle\right)=X$. By Fact [5] we can find a Borel set $B \subset \mathbb{R}$ containing $X$ and extensions $f_{n}^{*} \in \mathcal{C}(B)$ of the functions $f_{n}$ such that $S\left(\left\langle f_{n}^{*}\right\rangle\right)=B$. Then, there exists a $\xi<\omega_{1}$ such that $\left\langle f_{n}^{\xi}\right\rangle_{n<\omega}=\left\langle f_{n}^{*}\right\rangle_{n<\omega}$. Notice that $\bigcap_{\eta<\xi} P_{\eta} \subset S\left(\left\langle f_{n}^{\xi}\right\rangle_{n<\omega}\right)$, since otherwise $x_{\xi} \in X \backslash S\left(\left\langle f_{n}^{\xi}\right\rangle_{n<\omega}\right)$ contradicting $X=S\left(\left\langle f_{n}\right\rangle\right) \subset S\left(\left\langle f_{n}^{\xi}\right\rangle_{n<\omega}\right)$. Therefore, $\left\{x_{\zeta}: \xi \leq \zeta<\omega_{1}\right\} \subset P_{\xi}=\bigcup_{k<\omega} \bigcap_{k<n<\omega}\left\{x \in G_{\xi}:\left|f_{n}^{\xi}(x)\right| \leq \varepsilon_{n}^{\xi}\right\}$. Since the sequence $\left\langle\varepsilon_{n}^{\xi}\right\rangle_{n<\omega}$ works for all but countably many points of $X$, the existence of an appropriate sequence $\left\langle\varepsilon_{n}\right\rangle_{n<\omega}$ follows from an obvious fact that the class $\Sigma \Sigma^{*}$ is countably additive.

Thus, it is enough to show that the inductive construction is possible. The key point here is to prove that the intersection $\bigcap_{\eta<\xi} P_{\eta}$ is uncountable, so that we 
could have a chance to choose the next $x_{\xi}$. This will be done with the help of the families $\mathcal{G}_{\eta}$.

First notice that

$$
\text { for every } H \in \bigcup_{\eta<\xi} \mathcal{G}_{\eta} \text { there is a perfect set } H^{*} \subset H \cap \bigcap_{\eta<\xi} P_{\eta} \text {. }
$$

If $\xi$ is a successor ordinal, say $\xi=\alpha+1$, this is obvious: by the inductive assumption there is an $H^{*} \in \mathcal{G}_{\alpha}$ that is a subset of $H \cap \bigcap_{\eta \leq \alpha} P_{\eta}=H \cap \bigcap_{\eta<\xi} P_{\eta}$. So, assume that $\xi$ is a limit ordinal, and let $H \in \mathcal{G}_{\eta}$ for some $\eta<\xi$. Let $\eta=\eta_{0}<\eta_{1}<\cdots$ be such that $\xi=\bigcup_{n<\omega} \eta_{n}$. Define a family $\left\{H_{s}: s \in 2^{<\omega}\right\}$ by induction on the length of $s$. We put $H_{\emptyset}=H$ and, if for some $s \in 2^{n}$ the set $H_{s} \in \mathcal{G}_{\eta_{n}}$ is already defined, choose disjoint perfect subsets $H_{s^{\wedge} 0}$ and $H_{s^{\wedge} 1}$ from $\mathcal{G}_{\eta_{n+1}}$. The choice can be made by the inductive assumption (c) applied to the family $\mathcal{G}_{\eta_{n+1}}$ and disjoint clopen portions of $H_{s}$. Then $H^{*}=\bigcap_{n<\omega} \bigcup_{s \in 2^{n}} H_{s}$ is a perfect subset of $\bigcap_{\eta<\xi} P_{\eta}$.

Now, let $\mathcal{H}=\left\{H^{*}: H \in \bigcup_{\eta<\xi} \mathcal{G}_{\eta}\right\}=\left\{H_{k}: k<\omega\right\}$. We need to find a sequence $\left\langle\varepsilon_{n}^{\xi}\right\rangle_{n<\omega}$ for which

$$
H^{*} \cap P_{\xi} \text { contains a perfect subset for every } H^{*} \in \mathcal{H} \text {. }
$$

If $\bigcap_{\eta<\xi} P_{\eta} \not \subset S\left(\left\langle f_{n}^{\xi}\right\rangle_{n<\omega}\right)$, then $P_{\xi}=\mathfrak{C}$, and this is obvious. So, assume that $\bigcap_{n<\xi} P_{\eta} \subset S\left(\left\langle f_{n}^{\xi}\right\rangle_{n<\omega}\right)$. Then, by Proposition \&, for every $k<\omega$ there exists a sequence $\left\langle\hat{\varepsilon}_{n}^{k}>0: n<\omega\right\rangle$ such that $\sum_{n<\omega} \hat{\varepsilon}_{n}^{k}<\infty$ and there is a perfect subset $T_{k}$ of $\bigcap_{n<\omega}\left\{x \in H_{k}:\left|f_{n}(x)\right| \leq \hat{\varepsilon}_{n}^{k}\right\}$. Let $\varepsilon_{n}^{\xi}=\max \left\{\hat{\varepsilon}_{n}^{k}: k \leq k(n)\right\}$, where the sequence $k(0) \leq k(1) \leq \cdots$ converges to $\infty$ slowly enough that $\sum_{n<\omega} \varepsilon_{n}^{\xi}<\infty$. Then $T_{n} \subset P_{\xi}$ for every $n<\omega$ and the family

$$
\mathcal{G}_{\xi}=\left\{T_{n} \cap B \neq \emptyset: n<\omega \& B \in \mathcal{B}\right\}
$$

satisfies (c).

We would like to thank an anonymous referee for several suggestions that led to an improvement of the paper.

\section{REFERENCES}

[1] T. Bartoszyński, H. Judah, Set Theory, A K Peters, 1995. MR 1350295 (96k:03002)

[2] L. Bukovský, I. Recław, and M. Repický, Spaces not distinguishing pointwise and quasinormal convergence of real functions, Topology Appl. 41 (1991), 25-40. MR 1129696 (93b:54037)

[3] L. Bukovský, I. Recław, and M. Repický, Spaces not distinguishing convergences of real-valued functions, Topology Appl. 112 (2001), 13-40. MR 1815270 (2002e:54010)

[4] K. Ciesielski, Set Theory for the Working Mathematician, London Math. Soc. Stud. Texts 39, Cambridge Univ. Press, 1997. MR 1475462 (99c:04001)

[5] F. Galvin, A.W. Miller, $\gamma$-sets and other singular sets of real numbers, Topology Appl. 17 (1984), 145-155. MR 0738943 (85f:54011)

[6] J. Gerlits, Zs. Nagy, Some properties of $C(X)$, I, Topology Appl. 14 (1982), 151-161. MR 0667661 (84f:54021)

[7] W. Just, A.W. Miller, M. Scheepers, and P.J. Szeptycki, The combinatorics of open covers II, Topology Appl. 73 (1996), 241-266. MR 1419798 (98g:03115a)

[8] A.W. Miller, Special Subsets of the Real Line, in: Handbook of Set-Theoretic Topology (K. Kunen and J.E. Vaughan, eds.), North-Holland (1984), 201-233. MR 0776624 (86i:54037) 
[9] M. Repický, Porous sets and additivity of Lebesgue measure, Real Anal. Exchange 15(1) (1989/90), 282-298. MR 1042544 (91a:03098)

[10] W. Rudin, Real and Complex Analysis, McGraw-Hill Book Company, 1987. MR $0210528(35: 1420)$

Institute of Mathematics, Faculty of Sciences, P. J. Šafárik University, Jesenná 5, 04001 Košice, Slovakia

E-mail address: bukovsky@kosice.upjs.sk

Department of Mathematics, West Virginia University, Morgantown, West Virginia 26506-6310

E-mail address: K_Cies@math.wvu.edu

URL: http://www.math.wvu.edu/ ${ }^{\sim k c i e s}$ 